\title{
Monte Carlo Simulations of Intersubband Hole Relaxation in a GaAs/AlAs Quantum Well
}

\author{
R. W. KELSALL \\ Institute of Microwaves and Photonics, School of Electronic and Electrical Engineering, \\ University of Leeds, Leeds LS2 9JT, UK
}

\begin{abstract}
An ensemble Monte Carlo code has been developed for the simulation of hole relaxation processes in a GaAs/AlAs quantum well. The code includes a realistic k.p model of the valence subbands and corresponding wavefunctions. Intra- and inter-subband phonon scattering rates are calculated for polar and non-polar interactions via both optical and acoustic modes. The code is used to simulate the cooling of non-equilibrium photogenerated hole populations. A lifetime of $90 \mathrm{fs}$ is extracted for optical phonon mediated depopulation of the 4 th subband at $77 \mathrm{~K}$. By contrast, the 2 nd subband exhibits fast re-population, but slow de-population, with extracted lifetimes of up to $160 \mathrm{ps}$. The slow depopulation is attributed to the small energy separation of the 1 st and 2nd subbands (less than the optical phonon energy) and the large density of states in the 2nd subband off-zone-centre minimum.
\end{abstract}

Keywords: Monte Carlo, intersubband, quantum well

\section{INTRODUCTION}

Interest in intersubband transitions has been given new impetus by recent developments in "Quantum Cascade" or intersubband lasers for Far-InfraRed (FIR) operation [1]. Whilst the Quantum Cascade devices depend on electron intersubband transitions, $p$-type quantum well systems offer several advantages - high density of states, wide tunability and the possibility of normal incidence operation - and FIR emission has already been demonstrated in $p$-type germanium devices [2].
Design of FIR emitting devices requires detailed knowledge of the electronic bandstructure and transient carrier dynamics. The Monte Carlo method is an ideal simulation tool for these structures, since the time-dependent non-equilibrium carrier distribution function is intrinsically determined without recourse to any approximation, enabling accurate determination of intersubband lifetimes.

In this paper, an ensemble Monte Carlo code is used to simulate transient hole relaxation and energy loss phenomena in a GaAs/AlAs quantum 
well. The quantum confined valence bandstructure is calculated using an 8-band k.p scheme, to account for heavy-light hole mixing effects. The k.p wavefunctions are used to calculate holephonon scattering rates for the acoustic deformation potential, polar-optical, non-polar optical and piezoelectric interactions, via Fermi's Golden Rule. A total of 192 distinct intra- and intersubband scattering processes are included in the simulation. Degeneracy (Pauli exclusion) effects are also included. The $\mathbf{k}$-space trajectories of typically 100,000 particles are simulated, and cooling from a range of non-equilibrium carrier distributions is investigated.

\section{BANDSTRUCTURE AND SCATTERING RATES}

Figure 1 shows the in-plane hole energy dispersion for the lowest four subbands of a $100 \AA \mathrm{GaAs}$ quantum well. The bandstructure is calculated in the axial approximation, in which $\mathbf{k}$-space anisotropy is not resolved [3]. In practice, anisotropy in quantum wells is not as significant as that in the bulk GaAs valence bands. Figure 1 clearly shows the familiar distortion of the dispersion curves due to heavy-light hole mixing, including the presence of negative effective mass regions in the second and fourth subbands, and corresponding off-zonecentre minima. These minima give rise to VanHove singularities in the density of states, which result in strongly localised peaks in the scattering rates. When calculating the rates, we include a Lorentzian broadening function to model lifetime broadening effects which will 'smear out' these singularities [4]. The k.p calculation yields pairs of degnenerate eigenstates which have mixed spin parities. In this simulation, the spin states of individual carriers are not recorded, and the total rate for a given transition is obtained by summing the scattering rates into the two spin-degenerate states. The phonon scattering rates were calculated via Fermi's Golden Rule, assuming bulk-like phonon modes throughout. Figures 2 and 3 show

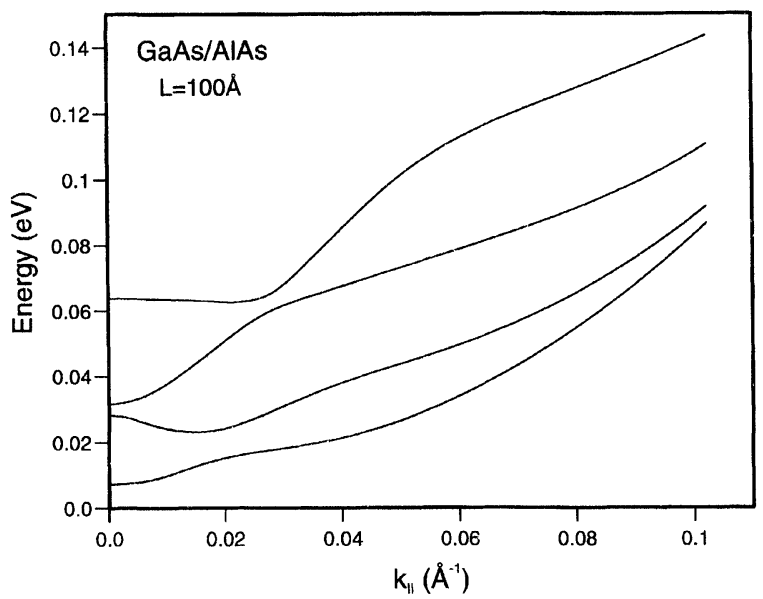

FIGURE 1 Energy vs. in-plane wavevector for the first four valence subbands in a $100 \AA \mathrm{GaAs} / \mathrm{AlAs}$ quantum well.

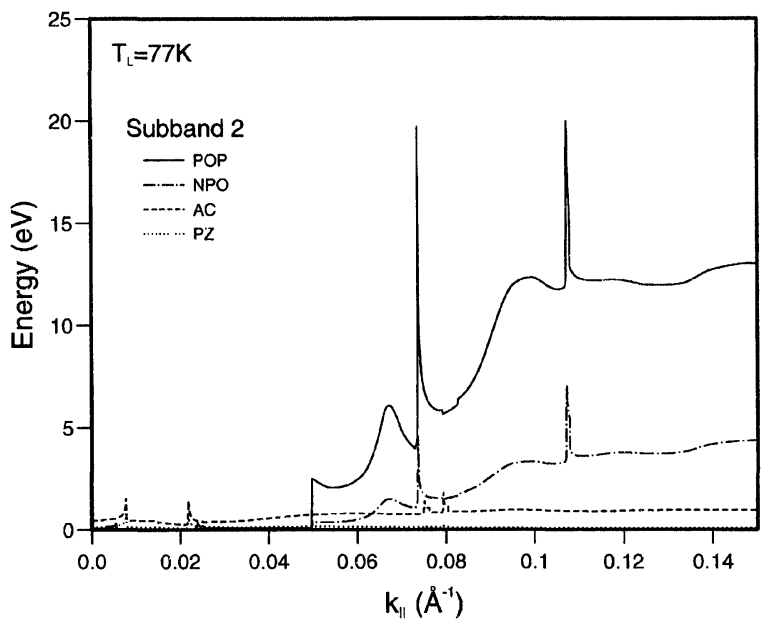

FIGURE 2 Phonon scattering rates for a hole in the 2nd subband, as a function of in-plane wavevector, for a lattice temperature of $T_{L}=77 \mathrm{~K}$, and a total hole density of $p=10^{11} \mathrm{~cm}^{-2}$. The graph shows contributions due to scattering via polar optical (POP), non-polar optical (NPO), acoustic deformation potential (AC) and piezoelectric (PZ) processes.

the total scattering rates for a hole resident in the 2nd and 4th subband, respectively, for a lattice temperature of $77 \mathrm{~K}$. In both cases, the dominant scattering processes are those due to polar optical phonons (Frölich interaction). However, for a carrier in the 2nd subband, optical phonon emission is only possible above a certain energy (wavevector) threshold, whereas in the 4th sub- 


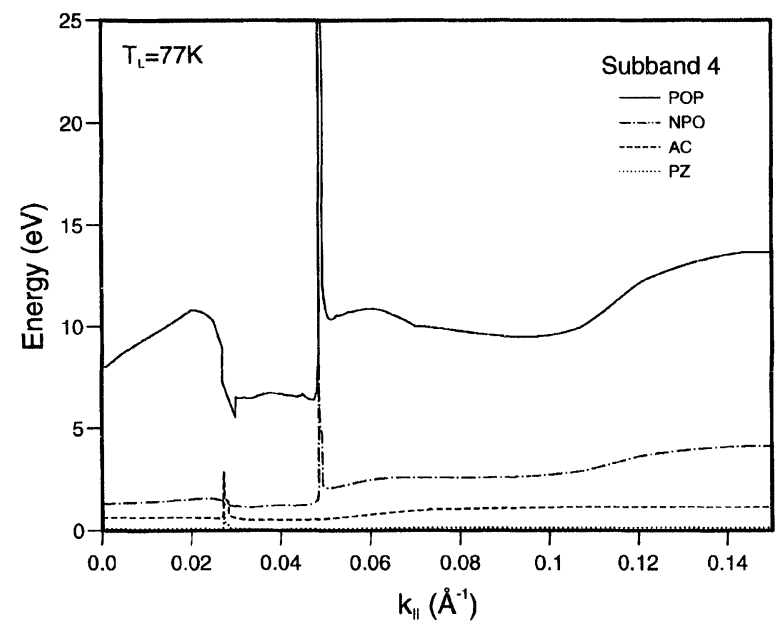

FIGURE 3 Phonon scattering rates for a hole in the 4th subband: details as for Figure 2 .

band, the 4-1 and 4--2 optical phonon emission processes are allowed at zero wavevector. Note that, in the valence bands, the deformation potential (non-polar) optical phonon interaction is not forbidden (as it is the conduction bands at the $\Gamma$ point), although the scattering rates are smaller than those for the polar interaction. Acoustic phonon scattering (modelled here by a single rate to represent the combined effects of the LA and TA modes) is only important at low carrier energies, and the piezoelectric interaction is weaker still, even at $77 \mathrm{~K}$. However, in our zerofield carrier cooling simulations, it is essential to include the acoustic phonon interactions, since they represent the principal energy relaxation processes for low energy carriers.

\section{HOLE RELAXATION SIMULATIONS}

The Monte Carlo model includes the four lowest energy subbands in the valence band quantum well of GaAs/AlAs. The subband energy dispersions, hole group velocities, and densities of states were tabulated as functions of in-plane wavevector. Similarly, the matrix elements for all permutations of intra- and inter-subband scattering via the relevant phonon modes were precalculated and supplied to the simulation as data. For the polar optical and piezoelectric processes, the matrix elements vary with the in-plane phonon wavevector $\left(q_{\|}\right)$, and hence with scattering angle. For polar optical scattering, we approximated this dependence by a $1 / q_{\|}^{2}$ variation for all cases. Similar analytic functions were derived to describe the angular dependence of piezoelectric scattering [5]. When calculating the new carrier states after an acoustic or piezoeletric phonon scattering event, the phonon energy $E_{\mathrm{AC}}$ was calculated using a linear phonon dispersion curve. For piezoelectric processes, a self consistent scheme was implemented to ensure that the choice of $q_{\|}$and the corresponding $E_{\mathrm{AC}}$ satisfied wavevector and energy conservation respectively, as well as following the appropriate probability distribution for $q_{\|}$. The optical phonon energy was assumed to be independent of wavevector.

The well was taken to be nominally undoped: no impurity scattering was included in the simulation. The results shown here are for simulations of an ensemble of 100,000 particles, whose energies, inplane wavevectors, and subband indices are monitored at each timestep. Degeneracy (Pauli exclusion) was included by compiling the hole distribution function in each subband at each timestep, and accounting for band-filling via a rejection technique. Since the initial energy distributions were highly non-equilibrium, a short timestep ( $1 \mathrm{fs}$ ) was chosen to ensure that bandfilling was not underestimated due to undersampling of the distribution. The simulations were carried out under zero field conditions: for this special case, the probability function which describes the distribution of free flight times can be inverted directly, without recourse to the selfscattering technique. This greatly increases the efficiency of the simulation.

As yet, there is very little experimental data on hole relaxation. Therefore, the aim of this initial work was to obtain information on the hole subband lifetimes and the many-subband cooling process, rather than attempting to simulate photoexcitation experiments. Such a direct comparison 
would also require inclusion of carrier-carrier interactions, which are not yet present in the simulation. In the following computer experiments, we assume that a highly non-equilibrium hole population has been photogenerated in an upper subband. The initial energy distribution is highly peaked at the assumed pump energy, with a narrow Gaussian distribution of carrier wavevectors to model Pauli exclusion within the subband. Two cases are presented below: firstly, photogeneration in the 4th subband, and secondly, into the 2nd subband; in both cases the pump energy corresponds to the subband energy minimum. The generated carrier density in both cases was $10^{11}$ $\mathrm{cm}^{-2}$.

Figure 4 shows the time dependence of the subband populations following carrier photogeneration in subband 4. As expected, subband 4 depopulates within $1 \mathrm{ps}$, due to fast polar optical phonon scattering into subbands 1 and 2 . Note that very few carriers are scattered into subband 3, since the energy separation between subbands 4 and 3 is less than the optical phonon energy. For $t<1 \mathrm{ps}$, subbands 1 and 2 are populated at approximately the same rate; however, for $t>1 \mathrm{ps}$, carriers begin to transfer from subband 2 to subband 1 . This transfer takes over $100 \mathrm{ps}$ to

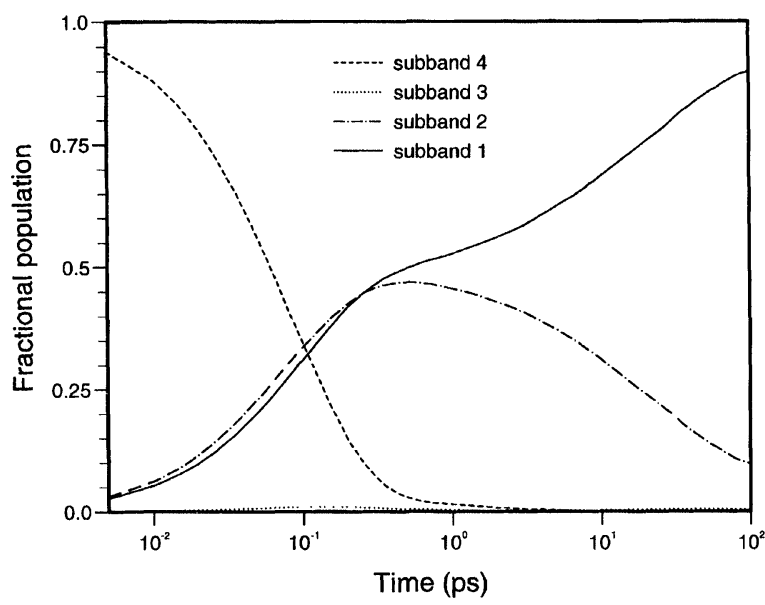

FIGURE 4 Simulated time dependence of the subband populations (normalised to unity) for an initial population photogenerated in subband 4: $T_{L}=77 \mathrm{~K}, p=10^{11} \mathrm{~cm}^{-2}$. complete, since the subband edge separation here, too, is less than the optical phonon energy, and most of the transitions are mediated via acoustic phonon emission (although there is some optical phonon emission from carriers located higher up the 2nd subband). One of the striking features about the population dynamics in this case is that the 2nd subband population rises to approximately 5 times its equilibrium value: in otherwords, this subband acts as a metastable carrier reservoir, which populates quickly, but depopulates much more slowly. This behaviour is, in part, due to the relative energy separations between subbands 4 and 2, and 2 and 1, but also due to the large density of states at the 2nd subband (offzone-centre) energy minimum.

We can use the simulation data to extract a value for the overall phonon scattering lifetime. Obviously, we already have information on lifetimes for individual phonon scattering processes, but in most photoluminescence experiments these processes cannot be separately resolved, and the extracted lifetime represents the combined effect of all participant scattering processs. Furthermore, the extracted result is modified by the exact nonequilibrium energy distribution of the carriers; a quantity which cannot readily be obtained by analytic methods, but which is inherently included in a Monte Carlo simulation. Not surprisingly, for such a complex system, we find that the lifetime approximation itself only holds within limited time ranges. For the initial cooling phase we estimate an overall depopulation lifetime for the 4th subband of approximately $\tau_{4}=90 \mathrm{fs}$. In contrast, in the latter phase of the simulation where carrier transfer from the 2nd to 1st subband occurs, we estimate a an overall depopulation lifetime for the 2nd subband of $\tau_{2}=90 \mathrm{ps}$.

Figure 5 shows the energy loss rate per carrier for the same simulation. The rate has also been resolved into components due to intra- and intersubband optical and acoustic phonon scattering, respectively. As expected, the initial rapid cooling is primarily due to optical phonon emission, but a crossover occurs after approximately 2 ps, where- 


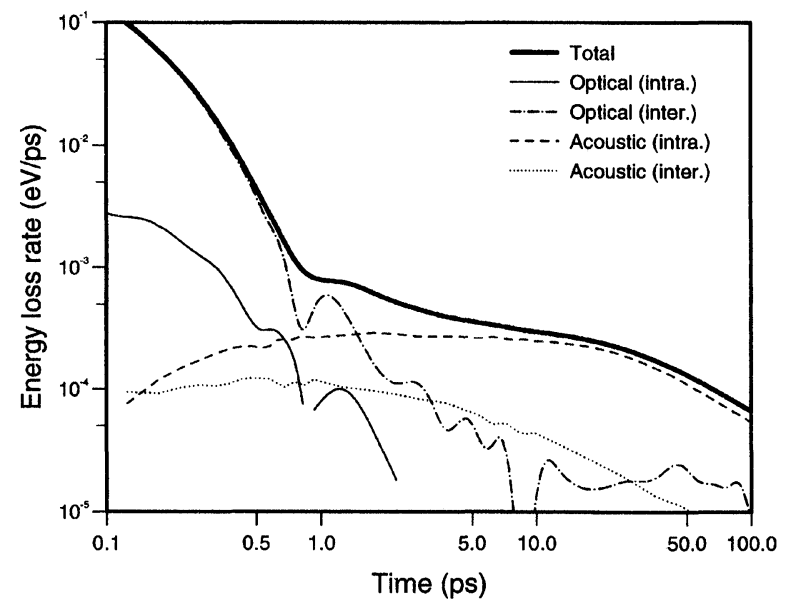

FIGURE 5 Total energy loss rate per hole, and energy loss rates due to intra- and inter subband optical and acoustic phonon processes: simulation conditions as for Figure 4.

upon acoustic scattering becomes the dominant energy loss mechanism.

Figure 6 shows the time dependence of the subband populations following carrier photogeneration in subband 2 . In this case, only two subbands participate in cooling, and we see a slow transfer of carriers from subband 2 to 1 . In the sub-picosecond phase, the transfer is characterised by a lifetime of approximately $\tau_{2}=4 \mathrm{ps}$, whereas in the $t>10 \mathrm{ps}$ phase, the extracted lifetime is $\tau_{2}=160 \mathrm{ps}$. Figure 7 shows the energy loss rates for this simulation. The total rate is actually negative for $t<2 \mathrm{ps}$. Examination of the simulation statistics reveal substantial (intrasubband) absorption of optical phonons by the 2 nd subband carriers in the initial phase of the simulation, followed by intersubband optical phonon emission to achieve transfer into subband 1 . The figure also shows some initial intersubband transfer by acoustic phonon emission, but this process is slower than the optical phonon absorption, and certainly much less effective in exchanging energy with the lattice. It is this intersubband optical phonon absorption which gives rise to the 4 ps depopulation lifetime - substantially longer than the lifetime in subband 4 , but still much shorter than typical acoustic phonon scattering lifetimes. For $t>2 \mathrm{ps,} \mathrm{a} \mathrm{net} \mathrm{energy} \mathrm{loss} \mathrm{is} \mathrm{observed,} \mathrm{and} \mathrm{is}$

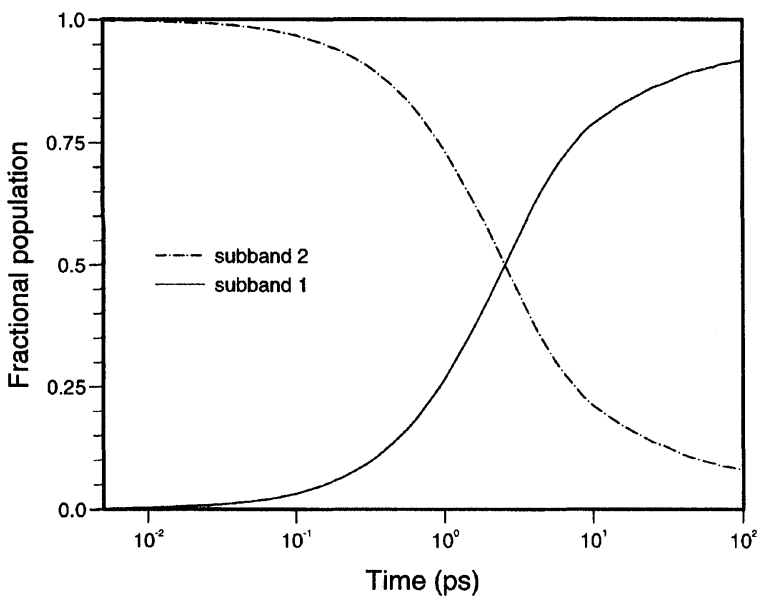

FIGURE 6 Simulated time dependence of the subband populations for an initial population photogenerated in subband 2: $T_{L}=77 \mathrm{~K}, p=10^{11} \mathrm{~cm}^{-2}$.

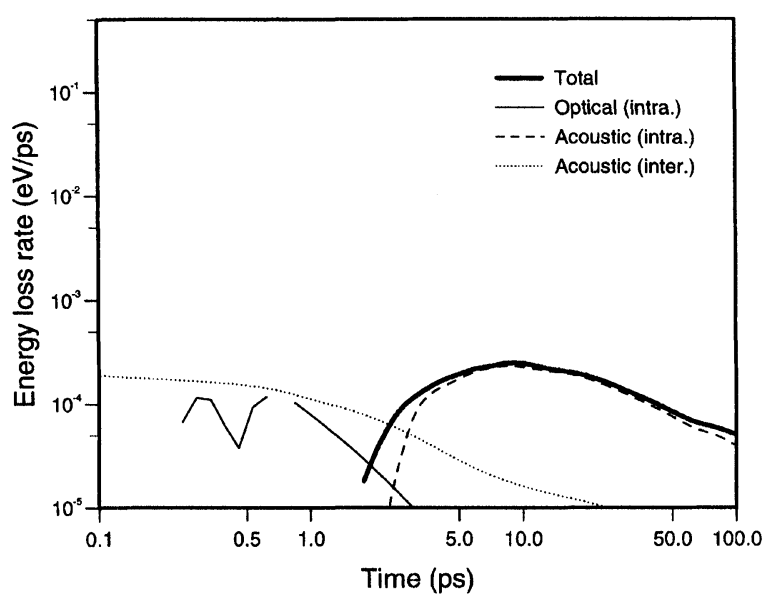

FIGURE 7 Total energy loss rate per hole, and energy loss rates due to intra- and inter subband optical and acoustic phonon processes: simulation conditions as for Figure 6 .

dominated by intrasubband acoustic phonon emission, as carriers relax within subband 1 . Comparing Figures 5 and 7 it can be seen that, for initial photogeneration in subband 4 , some intersubband $(2-1)$ optical phonon emission persists for $t>10 \mathrm{ps}$ whereas, for photogeneration in subband 2, this is not the case. This observation explains the difference in $\tau_{2}$ for the two simulations in the $t>10 \mathrm{ps}$ range: for initial photogeneration in subband 4 , the average carrier energy is 


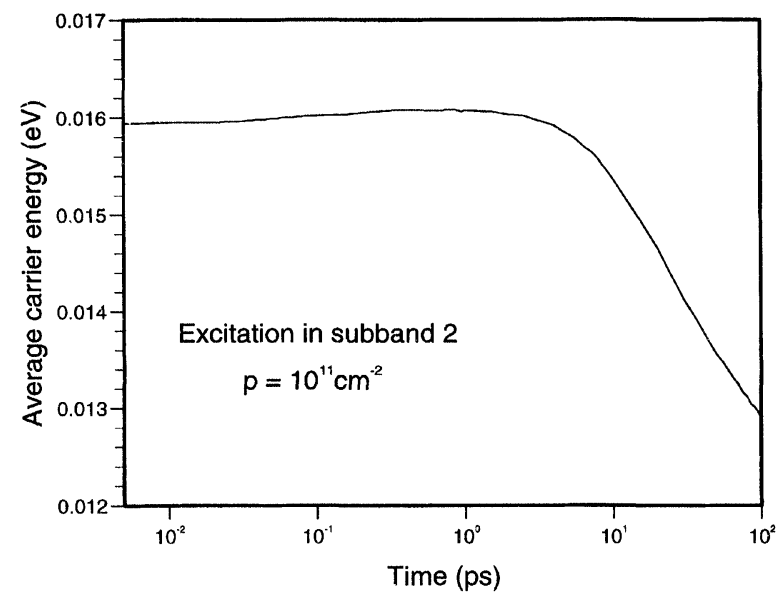

FIGURE 8 Average hole energy vs. time, for an initial population photogenerated in subband 2 .

higher, and hence some carriers are still able to emit optical phonons even after more than $10 \mathrm{ps}$ of cooling time; thus $\tau_{2}$ is enhanced by optical phonon scattering. On the other hand, for photogeneration in subband 2 , the average carrier energy is lower, and $\tau_{2}(t>10 \mathrm{ps})$ is entirely acoustic-phonon limited. Figure 8 shows the time dependence of the overall average carrier energy for this simulation. A small increase in average energy is indeed observed throughout the range $t<5 \mathrm{ps}$, with a clear decrease thereafter. Again, this implies that the second subband acts as a bottleneck to the hole cooling process. Clearly, this conclusion may be affected significantly by carrier-carrier scattering, dependent on the extent to which intersubband inter-carrier processes will redistribute both energy and population. Calculations for electrons in quantum wells show that such processes occur over a range of timescales of the order $10^{0}$ ps [6].

\section{CONCLUSIONS}

An ensemble Monte Carlo code has been developed which includes a realistic description of the bandstructure, density of states, and phonon scattering rates for holes in a GaAs/AlAs quantum well.
Simulations of intersubband hole relaxation indicate that the 2 nd valence subband of the quantum well acts as a bottleneck for hole cooling, due to both the small 2-1 subband energy separation and the large density of states in the off-zone centre minimum. Consequently, a 2nd subband lifetime as long as $160 \mathrm{ps}$ is predicted at $77 \mathrm{~K}$, compared to a lifetime of $90 \mathrm{fs}$ for the 4th subband. The large difference in the re-population and de-population rates of the 2nd subband can result in the accumulation of large excess carrier populations; which implies that Far-Infra-Red photon emission may be attainable between the 1st and 2nd subbands under suitable excitation conditions.

\section{Acknowledgment}

This work was initially begun by the author at the University of Durham, UK, with support from the UK Engineering and Physical Sciences Research Council. Advice and guidance from Prof. R. A. Abram at Durham is gratefully acknowledged.

\section{References}

[1] Faist, J., Capasso, F., Sivco, D. L., Sirtori, C., Hutchinson, A. L. and Cho, A. Y. (1994). "Quantum cascade laser", Science, 264, 553.

[2] Heiss, W., Unterrainer, K., Gornik, E., Hansen, W. L. and Halter, E. E. (1994). "Influence of impurity absorption on Germanium hot-hole laser spectra", Semicond. Sci. Technol., 5, SS638.

[3] Eppenga, R., Schuurmans, M. F. H. and Colak, S. "New k.p theory for GaAs/AlGAAs type quantum wells", Phys. Rev. B., 36, 1554.

[4] Kelsall, R. W., Wood, A. C. G. and Abram, R. A. (1991). "Phonon scattering and mobility of holes in a GaAs/AlAs quantum well", Semicond. Sci. Technol., 6, 841.

[5] Kelsall, R. W. (1989). Ph.D. Thesis, University of Durham.

[6] Goodnick, S. M. and Lugli, P. (1988). "Effect of electronelectron scattering on non-equilibrium transport in quantum well system", Phys. Rev. B., 37, 2578.

\section{Author Biography}

Robert W. Kelsall was born in Rotherham, England in 1964. He received the B.Sc. and Ph.D. degrees from the University of Durham, 
UK, in 1985 and 1989 respectively; the latter for research in Monte Carlo modelling of electronic transport in GaAs quantum wells.

From 1989 to 1993 he worked as a research assistant, developing self-consistent Monte Carlo simulations of HEMTs and multilayer MOSFETs. $\mathrm{He}$ was appointed to a lectureship at the Uni- versity of Leeds, UK in 1993, and is a member of the newly formed Institute of Microwaves and Photonics at Leeds, where he is working on semiconductor device simulations for hot-carrier degradation studies in MOSFETs, quantum confinement effects in HEMTs, and far-infra-red intersubband lasers. 

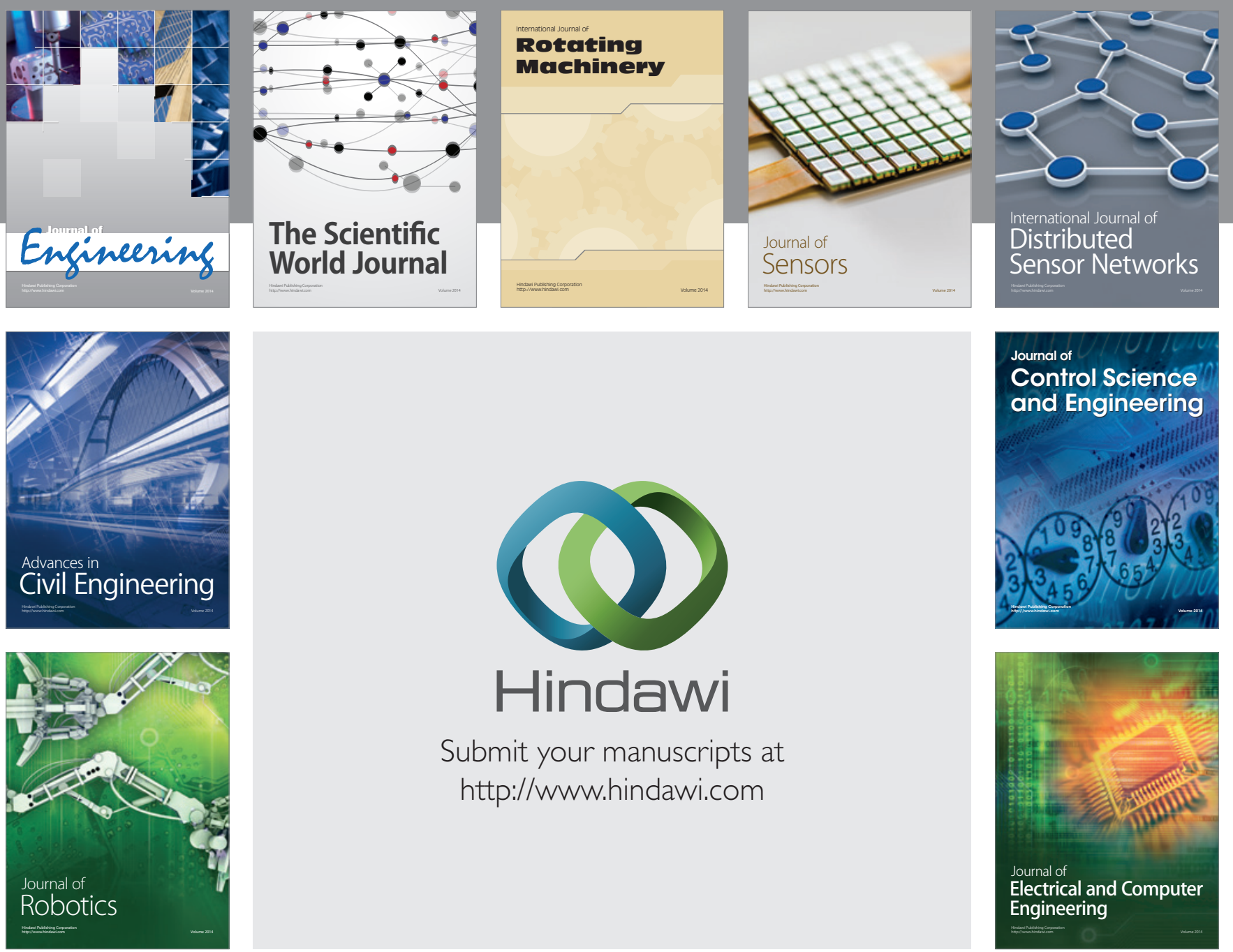

Submit your manuscripts at

http://www.hindawi.com
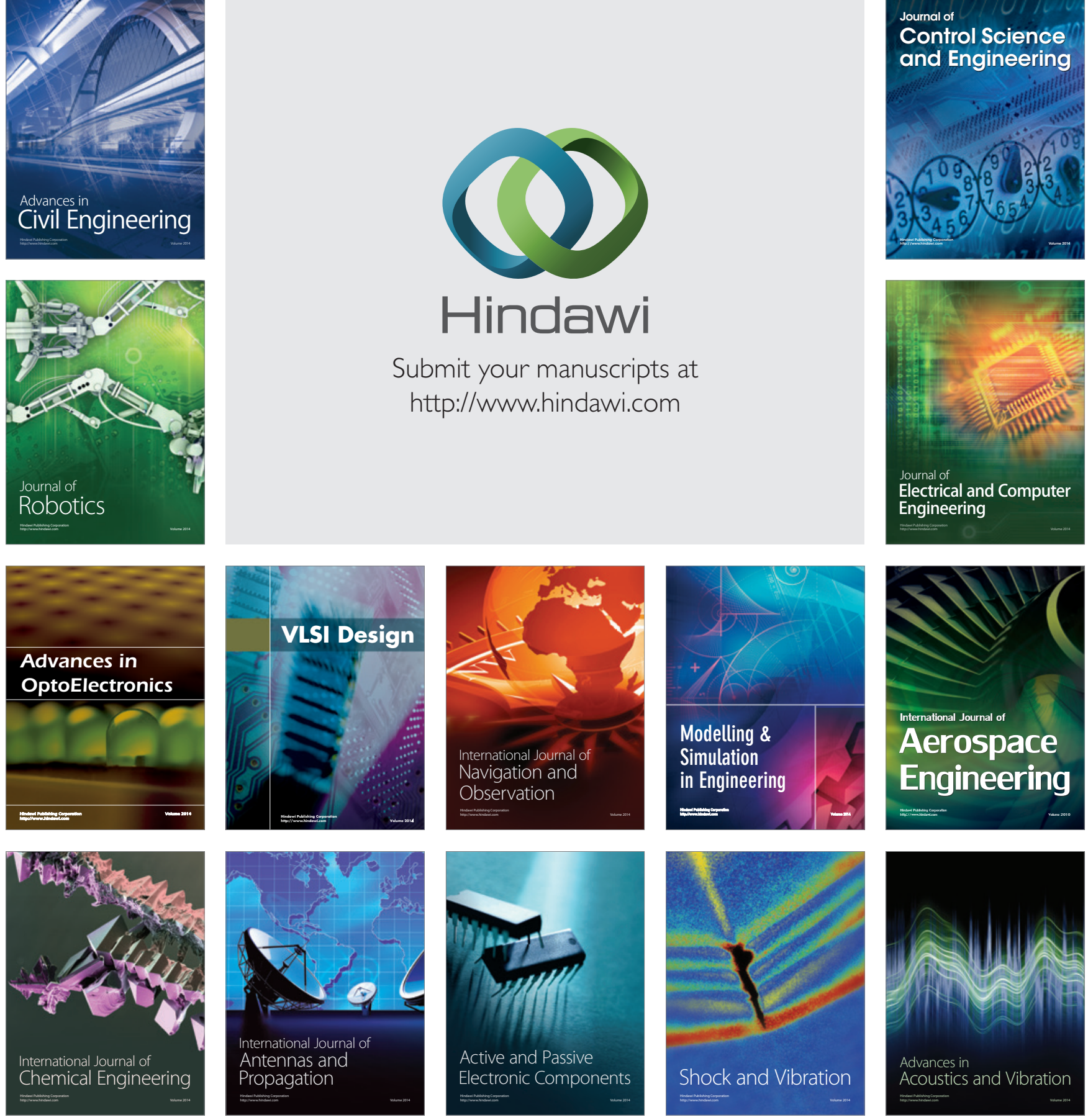